\title{
Antisynchronous spike patterns in dye coupled GABAergic feedback neurons in the brain of the honeybee Apis mellifera
}

\section{[version 1; peer review: 1 approved]}

\author{
Nora Vanessa de Camp \\ Department of Behavioral Physiology, Humboldt University Berlin, Berlin, 10115, Germany
}

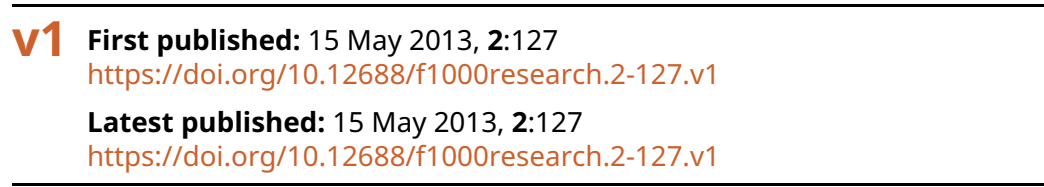

\section{Abstract}

Intracellular recordings in GABAergic feedback neurons in the mushroom body of the honey bee brain revealed patterns of alternating neural activity. The recorded neurons have been identified via iontophoretic injection of Neurobiotin. The staining of groups of cells indicated dye coupling on the basis of gap junctions. The corresponding spike activity revealed spikes with different but overall comparatively low amplitudes ("spikelets"). The assumption of axoaxonal gap junctions would explain the staining of clusters of feedback neurons, alternating unit activity as well as spikelets with low amplitude. If a neuron in the electrically coupled network fires at a lower than maximal firing rate with respect to the refractory period, it will become more susceptible to laterally incoming spikes of neighbouring feedback neurons. In succession, the respective cell can be fully overtaken by neighbouring spike activity. On the one hand this proposed mechanism could lead to highly synchronous spike activity of a huge number of inhibitory synapses in the mushroom body calyces. On the other hand, the mechanism of laterally spreading activity could act like an amplifier. Additionally, the anatomical properties of Protocerebro Calycal Tract (PCT) feedback neurons could account for a reset function in order to allow for the changing activity profiles of the coupled cells. The laterally incoming potential would run in an antero- and retrograde direction. This could in principle lead to backpropagating inhibition of neighbouring PCT neurons and therefore a reset of one gating cycle. The proposed resulting oscillatory pattern of PCT activity has already been described in the literature and is confirmed by the physiological results, presented here.

\section{Keywords}

Honeybee, spike patterns, GABA, GAD, protocerebro calycal tract, spike patterns, sychrony, antisynchrony, feedback neurons

\section{Peer review discontinued \\ Peer review at F1000Research is author- driven. Currently no reviewers are being invited. What does this mean?}

Approval Status
version 1
Heidelberg, Germany reports and responses or comments on the
article can be found at the end of the article.


Corresponding author: Nora Vanessa de Camp (nora.de.camp@hu-berlin.de)

Competing interests: No competing interests were disclosed.

Grant information: The author(s) declared that no grants were involved in supporting this work.

Copyright: $\odot 2013$ de Camp NV. This is an open access article distributed under the terms of the Creative Commons Attribution License, which permits unrestricted use, distribution, and reproduction in any medium, provided the original work is properly cited. Data associated with the article are available under the terms of the Creative Commons Zero "No rights reserved" data waiver (CC0 1.0 Public domain dedication).

How to cite this article: de Camp NV. Antisynchronous spike patterns in dye coupled GABAergic feedback neurons in the brain of the honeybee Apis mellifera [version 1; peer review: 1 approved] F1000Research 2013, 2:127

https://doi.org/10.12688/f1000research.2-127.v1

First published: 15 May 2013, 2:127 https://doi.org/10.12688/f1000research.2-127.v1 


\section{Introduction}

Synchrony of spike trains in the brain has a major function for the strengthening of synaptic contacts and the formation of phenomena such as long term potentiation (LTP) (reviewed by Brown et al. ${ }^{1}$ ). Synchrony is one proposed mechanism for the formation of a neural syntax and inhibitory interneurons are believed to play an important role in the underlying cell assembly formation ${ }^{2}$. Gap junction-mediated electrical coupling has been shown to be a potential source of not only synchrony but also asynchrony ${ }^{3}$. Apart from mere synchrony effects, axoaxonal gap junctions in blowfly visual interneurons have been proposed to introduce a linear interpolation system between coupled neurons in order to visually extract the axis of rotation $^{4}$. Gap junctions between pyramidal neurons in the rat hippocampus are suggested to contribute to sharp wave/ripple local field potentials ${ }^{5}$. In the human brain, gap junction-mediated quantum entanglement of microtubules has been proposed as a mechanism for very fast states of conscious perception ${ }^{6}$. The mushroom bodies are intensively studied neuropiles of the insect brain involved with learning and memory ${ }^{7,8}$. The mushroom body in each brain hemisphere of the honeybee consists of about 170000 intrinsic Kenyon cells ${ }^{9}$. The dentritic arborizations of these cells form the cup shaped calyces which are characterized as the main input region of the mushroom body ${ }^{10}$. Each calyx of a mushroom body is further subdivided into a lip, a collar and a basal ring neuropile ${ }^{11}$. The lip receives mainly olfactory input, the collar receives visual input and the basal ring receives mixed modality sensory input ${ }^{11}$. The Kenyon cell axons are bundled and form the two peduncles that connect the input region of the mushroom body, the two calyces, with the output regions, the alpha and beta lobes $^{12}$ (Figures 1, 2). The modality-specific topographic organization of Kenyon cells in the calycal regions is maintained throughout the mushroom body and causes a layered pattern of the lobes ${ }^{11,13}$. Feedback neurons are apparent in the mushroom bodies of a great variety of insect species (ant (Formica rufa, F. pratensis) ${ }^{14}$, grasshopper (Acheta domesticus) ${ }^{15,16}$, fly (Musca domestica) ${ }^{17}$ and bees (Apis mellifera $\left.)^{11,18}\right)$.

Protocerebro Calycal Tract (PCT) neurons are anatomically described feedback neurons in the honeybee brain, which receive inputs from the lobes and calycal regions ${ }^{19}$ and form spiny endings in the calyces which are interpreted as post-synapses ${ }^{10,20}$. It has been shown that PCT neurons do not exclusively connect corresponding regions between the calyces and lobes but also layers with different sensory modality in the mushroom body ${ }^{20}$. Due to the location of the somata in the lateral protocerebral lobe, a ventral (A3-v) and a dorsal (A3-d) cluster of feedback neurons can be distinguished ${ }^{13}$. The somata of the A3-v cluster are located in the anterior, ventral, lateral protocerebral lobe. The A3-d somata are located in the vicinity, slightly dorsal of the A3-v soma cluster ${ }^{13}$ (Figure 1 ). The primary neurites of both tracts run separately through the protocerebral lobe and cross half way to

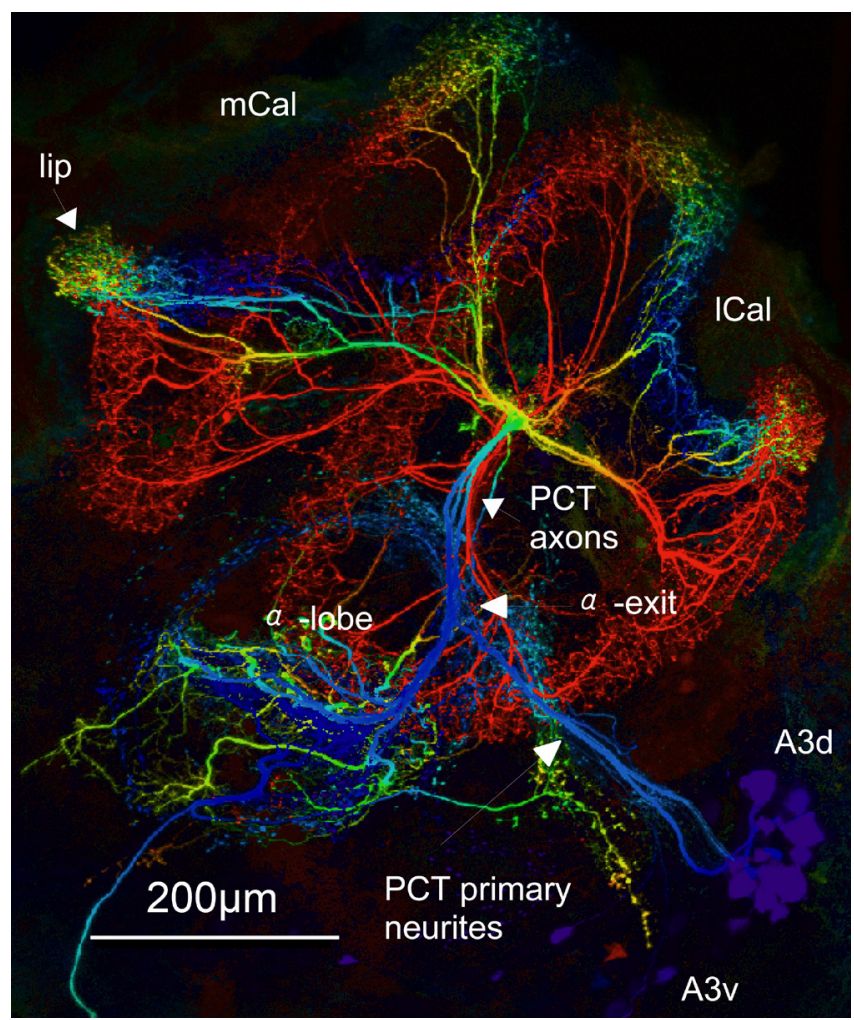

Figure 1 Spatial distribution of fibres in the Protocerebro Calycal Tract (PCT) in the honeybee brain. The primary neurite runs from the lateral protocerebral lobe towards the lateral margin of the alpha lobe (alpha-exit) in one hemisphere of the bee brain. Reaching the alpha lobe, the PCT bifurcates, sending dendritic branches to both the alpha lobe and parallel axonal projections towards the calyces. The lateral and median calyces ( $\mathrm{ICal}, \mathrm{mCal})$ are intensely innervated by PCT neurons, especially the lip region. The resulting cup shaped dendritic innervation areas are seen on top of the figure. Depth is color coded, the ventral and dorsal somata cluster (A3v, A3d) are superficial. Warm colors indicate a deep layer (ventral), whereas cool colors indicate a superficial (dorsal) position in the brain (blue, green, yellow, red), with blue most superficial and red for the deepest layers. Confocal scanning with a factor of 100x magnification.

the alpha lobe ${ }^{13}$. Both tracts invade the alpha lobe at its lateral border, the A3-d approximately $20 \mu \mathrm{m}$ more anterior than the $A 3-v^{13}$. The A3-v tract is identical to the anterior lateral protocerebral tract (a.l.p.t.), described by Mobbs ${ }^{11,13}$. The cells of the A3-v branch dichotomously in the protocerebral lobe, dorsolaterally to the alpha lobe. One branch continues in the PCT, the other branch benches ventrally and enters the alpha lobe at its lateral margin, the alpha exit point ${ }^{11}$ (Figure 1). The whole bee brain is shown in Figure 3B. The PCT neurons form layered innervation patterns in the alpha lobe. Some branches run posterior to the alpha lobe, in parallel to the Kenyon cells, towards the beta lobe (orange dendrites in Figure 2). The branch towards the beta lobe is more prominent for A3-d PCT neurons. The dendrites run via the alpha-lobe-to-beta-lobe-tract (a-b.t. ${ }^{13}$ ) and invade the beta lobe at its lateral margin at a depth of approximately $160 \mu \mathrm{m}$. 


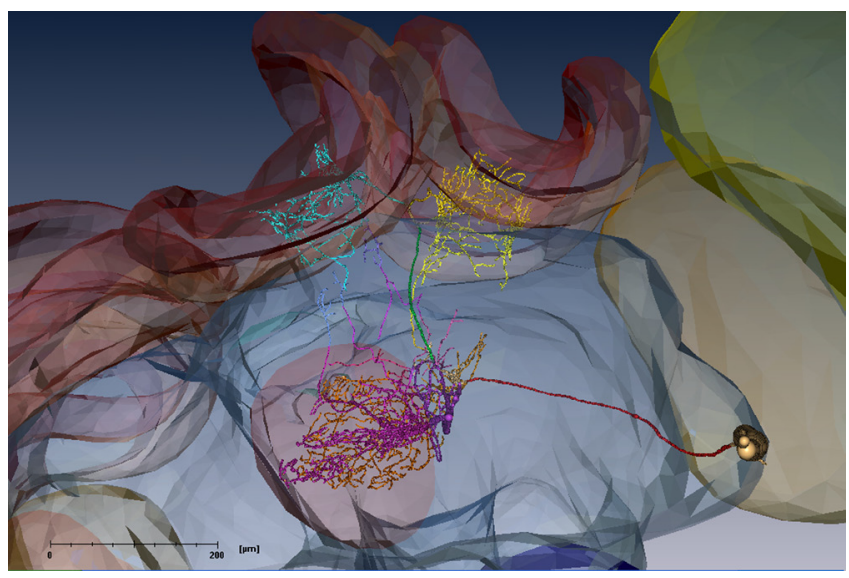

Figure 2 3D reconstruction of a single Protocerebro Calycal Tract (PCT) neuron. Unlike most PCT neurons stained in the examples beforehand, this PCT neuron has no dendrites in the calyces. The dendritic arborizations are restricted to the pedunculi (cyan and yellow). Additionally, the neuron has large dentritic trees in the alpha (magenta) and beta (orange) lobes.

The second branch of A3-v PCT neurons runs via the inner ring tract (i.r.t. ${ }^{11}$ ) towards the ipsilateral calyces. A3-v-PCTs innervate the entire calycal neuropile. Nevertheless, the highest density of dendritic branches of A3-v PCT neurons has been observed in a small region between the collar and basal ring (dorsal basal ring, $\left.\mathrm{d}-\mathrm{br},{ }^{13}\right)$. The lip and the basal ring neuropiles are also common targets of A3-v innervation. In the lobe region postsynaptic spines have been described ${ }^{13}$.

The majority of PCT neurons are GABAergic ${ }^{18}$ and in locusts, GABAergic interconnections have been shown between the lobes $^{21}$. The occurrence of post- and presynaptic GABAergic terminals in the lobes has been confirmed in the bee brain ${ }^{22}$. For the A3-v PCT neurons with dendritic arborizations in the basal ring as well as pedunculus neuropile, claw-like dendritic endings have been observed. In contrast, the majority of dendritic endings for A3-v neurons that arborize in the basal ring reveal a round, bleb like character ${ }^{13}$. The physiological properties of PCT neurons are less uniform. The spontaneous firing rate is between 0 and $45 \mathrm{~Hz}^{20}$ or between 0 and $24 \mathrm{~Hz}$ and bursts occur frequently ${ }^{23}$. The spontaneous activity is not homogenous with respect to the spike distribution. The intracellular recorded spikes at the lateral margin of the alpha-lobe are lacking clear after-hyperpolarization "spikelets" ${ }^{23}$. Grünewald ${ }^{23}$ observed phasic-tonic excitatory responses $(3.3-48 \mathrm{~Hz}$ above the respective spontaneous activity) to odorant stimulation, with a $500 \mathrm{~ms}$ phasic period after a latency of 75 to $128 \mathrm{~ms}$. $22 \%$ of feedback neurons showed excitatory off-responses with a short duration but longer latency than on-responses ${ }^{23}$. Typically, their response to stimuli is excitatory with an extended latency between the stimulus and the increase in spike rate (25-70 ms for light stimuli, up to $400 \mathrm{~ms}$ for odorants $)^{20}$.
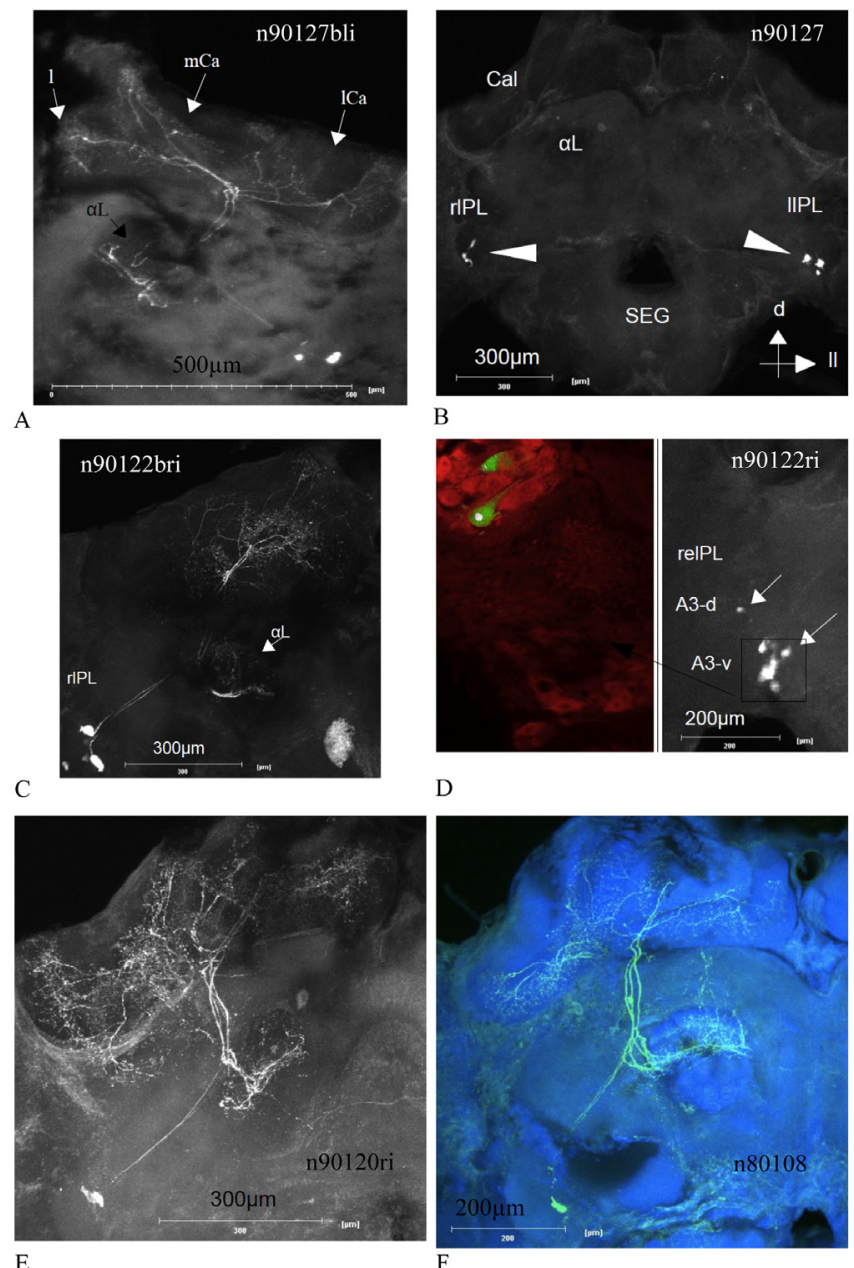

Figure 3 Intracellular stainings of Protocerebro Calycal Tract (PCT) neurons (100x magnification). The images are anatomical correlates for the recordings presented in Figure 4. Identification of PCT neurons was achieved by either full staining (panels $\mathbf{A}, \mathbf{C}, \mathbf{E}$ and $\mathbf{F}$ ) or characteristic soma clusters (panels $\mathbf{B}$ and $\mathbf{D}$ ). Panel $\mathbf{B}$ gives an overview of a whole bee brain, with right and left lateral protocerebral lobe (rIPL, IIPL), subesophageal ganglion (SEG), alpha lobe $(\alpha-L)$ and calyces $(\mathrm{Cal})$. The arrowheads indicate the PCT soma cluster. The arrows indicate dorsal (d) and lateral left (II) direction. In panel $\mathrm{A}$, the median and lateral Calyx $(\mathrm{mCa}, \mathrm{ICa})$ as well as the lip region $(I)$ are indicated for one brain hemisphere. The inset in panel $D$ corresponds to the colocalization between some intracellularly stained PCT somata of the ventral cluster (A3v). The anti-GABA staining is color coded in red, a colocalization with the intracellular staining (white) is indicated in green (200x magnification). The region of the nucleus does not contain any GABA staining and is therefore seen as small white spot (panel D, inset).

Nevertheless, inhibitory responses to odor stimuli have been observed $^{20}$. PCT neurons respond to different sensory modalities, for example visual, olfactory or tactile stimulation but not movement $\mathrm{t}^{20}$. The responses of PCT neurons to odor stimulation are more different between neurons for the same odor than in the same neuron for different odorants ${ }^{23}$. The relative spike rate is decreased after one trial odor conditioning in contrast 
to sensitisation ${ }^{23} .92 \%$ of the PCT neurons show excitatory responses after stimulation of the ipsilateral antenna with sugar solution $^{23}$. Gronenberg ${ }^{20}$ observed after-effects, which are responses with latencies longer than $500 \mathrm{~ms}$ (up to $30 \mathrm{~s}$ after stimulation has been observed). Interestingly, Gronenberg ${ }^{20} \mathrm{de}-$ scribed complex spike characteristics in an individual feedback neuron, which completely changed its response characteristics regarding latency, spike frequency and response type (inhibitory vs. excitatory) during the experiment.

This article aims to investigate the functional relationship between anatomy and physiology in PCT GABAergic feedback neurons in the mushroom body of the bee brain. The experimental results presented here point towards the occurrence of electric coupling between PCT neurons. This hypothesis will be discussed in the context of recent findings and earlier results.

\section{Materials and methods}

\section{Ethics statement}

No specific permissions were required for the experiments with invertebrates (honey bees), owned by Freie Universität Berlin.

\section{Animals and preparation}

Forager honey bees (Apis mellifera, $n=7$ ) were caught from a winter indoor flight room with small glass vessels. The bees were chilled on ice and fixed in small plastic holders with a slit for the neck. The head was additionally stabilized towards the holder with a small piece of plastic sheet, which was adjusted with beeswax. The body was held in place with wax inside the recording chamber to prevent turning movement of the body against the head. After a recovery period of 30 minutes in a dark and moist chamber, the bees were fed to saturation with $1.25 \mathrm{M}$ sucrose solution. At least one hour later the bees were operated on. The antennae were fixed with Eicosan (Sigma-Aldrich, Germany) to the head capsule. The mandibular muscles were cut and the mandibles fixed with Eicosan to the recording chamber in order to prevent movement artefacts. A trapezoid shaped cut at the clypeus between antennal joints and mandibles allowed for the removal of the oesophagus. The reference electrode was placed in the median ocellus. The head capsule was cut between the ocelli, compound eyes and antennae (without damaging the antennal nerves) in order to make the brain accessible. Trachea on the surface of the brain and mandibular glands were removed with a fine forceps. The bees' abdomen was pressed with a piece of wax (Boxing wax sticks, Kerr Corporation, United States) in order to prevent pumping movements. A two component silicon sealant (Kwik-Sil, World Precision Instruments, Inc., United States) was used to build a fluid barrier and stabilization ring at the border of the window in the head capsule. After polymerization of the two component silicon ring, the single, fluid component $\mathrm{A}$ was used as surface medium on top of the brain tissue in order to prevent drying.
Intracellular recordings and stains

Borosilicate glass capillaries with filament (Hilgenberg, Germany) were used for intracellular recordings (outer diameter $1 \mathrm{~mm}$, length $75 \mathrm{~mm}$, wall thickness $0.21 \mathrm{~mm}$ ). A laser puller (P-2000, Sutter Instrument Co., United States) was used for capillary production. The resulting electrode resistance was between 100 and $300 \mathrm{Mohm}$, depending on the dye filling. The tip of the capillary was filled with a $5 \%$ solution of Neurobiotin Tracer (Vector Laboratories Inc., United States) in $0.2 \mathrm{M}$ or $1 \mathrm{M} \mathrm{K}$-Acetate. The electrode was inserted with a micromanipulator under visual control on the lateral margin of the alpha lobe towards the lateral protocerebral lobe neuropile at three o'clock for the left alpha lobe and nine o'clock for the right one. The onset of tissue contact to the glass capillary was indicated by the silencing of an acoustic signal (Intra767, Electrometer, World Precision Instruments, United States). From the offset of the acoustic signal for the touching point of the tissue surface, the electrode was slowly driven into the brain until a depth of 60 to $160 \mu \mathrm{m}$ was reached. Analogue data were visualized with an oscilloscope (630, Voltcraft, United Kingdom). For data acquisition, the combination of Spike2 software and the corresponding analogue to digital converter (Micro 1401 MKII, Cambridge Electronic Design, CED) was used with a sampling rate of $20000 \mathrm{~Hz}$. Intracellular stainings (Neurobiotin) was achieved by iontophoresis with a depolarizing current of 3-4 $\mathrm{nA}$ for a duration of 10-20 minutes. The staining procedure was observed and interrupted in the case of unstable recordings.

\section{Multimodal stimulation of feedback neurons}

Spike2 was used to control a relais card (or8, bmcm, Germany) which was connected to different odor valves (The Lee Company, United States). A continuously presented air stream $(1.5 \mathrm{~m} / \mathrm{s})$ without odorant was applied throughout the experiments. During the presentation of an odor the valve switched from the neutral airstream to an odorant laden airstream. The strength of the air stream was constant throughout the experiments. Each odorant supplier was filled with a small piece of filter paper, soaked with $2 \mu$ of one odorant (orange oil, carnation oil, linalool, limonene, cineole, geraniol, hexanol, nonanol, hexanal, heptanal, octanal, 2-nonanone or 2-octanone). Blue, green, white and UV light were presented manually with an LED device. Both the olfactory and the visual stimulation took place for a duration of $4 \mathrm{~s}$. Tactile stimulation was induced by a dry toothpick towards the antennae. For sugar stimulation the toothpick was soaked in $1.25 \mathrm{M}$ sugar solution. The sugar stimulation consisted of one short touch towards the antennae.

\section{Immunohistochemistry}

The post iontophoresis circulation time in the living bee took at least $3 \mathrm{~h}$ or overnight in a dark and moist chamber with a temperature of $20^{\circ} \mathrm{C}$. Afterwards the Kwik Sil was removed from the brain. The pre-fixed brain was dissected from the head capsule and thereafter carefully lifted from the silicon layer. 
The brain in the head capsule was pre-fixed in $4 \%$ paraformaldehyde (PFA, Electron Microscopy Science, United States) in PBS (NaCl: $137 \mathrm{mM}, \mathrm{KCl}: 2.7 \mathrm{mM}, \mathrm{Na}_{2} \mathrm{HPO}_{4}: 8 \mathrm{mM}$, $\left.\mathrm{KH}_{2} \mathrm{PO}_{4}: 1.4 \mathrm{mM}, \mathrm{pH}: 7.2\right)$ for 30 minutes. The brain was carefully removed from the head capsule, the silicon and surrounding tissues as well as the trachea. The brain was retained in PFA solution during this cleaning procedure. Each brain was separately fixed for an additional $4-6 \mathrm{~h}$ in $500 \mu \mathrm{l}$ 4\% PFA solution in a small glass vessel. The fixed brain was dehydrated in an alcohol series on a shaker at room temperature (at 50\%, $70 \%, 90 \%, 99 \%$ and then 3 times at 100\% ethanol and step wise back to $50 \%$ ethanol again, each step taking 10 minutes). Subsequently the brain was washed in PBS on a shaker for 10 minutes twice at room temperature and incubated in $1 \%$ TX (TritonX, Sigma, Germany) in PBS for $2 \mathrm{~h}$ on a shaker at room temperature. The Streptavidin conjugation (Cy5, dianova, Germany) took place over night but not longer than $20 \mathrm{~h}$ at $4^{\circ} \mathrm{C}$ on a shaker in a Streptavidin Cy5 solution 1:1000 in PBS plus $1 \mu \mathrm{l}$ sodium acid (saturated stock solution). For confocal microscopy $1 \mu \mathrm{l}$ of a 5\% Lucifer Yellow (Invitrogen, Germany) solution in Aqua Dest (custom made) was added to achieve a background staining of the brain neuropile. From this step on the preparation was light sensitive and needed to be shielded from bleaching. Unbound Streptavidin was removed from the brain via 6 washing cycles in PBS at room temperature on a shaker (brief washing for 10 minutes, 20 minutes, two times 30 minutes and one time 60 minutes). For dehydration, an ascending alcohol series was used $(50 \%, 70 \%, 90 \%, 99 \%$, and twice at $100 \%$, each step for 10 minutes on a shaker at room temperature). Afterwards the brain was stored and cleared (tissue transparency was required for confocal microscopy) either in methylsalicylate (Roth, Germany) or in a 2:1 mixture of benzylbenzoate and benzyl alcohol (Sigma, Germany). Prior to storage in methylsalicylate, the brains were washed twice for 15 minutes at room temperature on a shaker or at least twice for 30 minutes in the benzylbenzoate benzylalcohol mixture. In order to apply an antibody staining in addition to the intracellular staining, the brains were washed in a descending ethanol series, to bring them back into PBS (as described before, twice at $100 \%$, then $99 \%, 90 \%, 70 \%$, and $50 \%$ ethanol and PBS, each step for 10 minutes on a shaker at room temperature). The brains were dried on a piece of absorbent tissue and subsequently embedded in agarose gel $(0.3 \mathrm{~g}$ agarose in $5 \mathrm{ml} \mathrm{PBS})$. A Leica vibratome (VT 1000 S, Germany) was used to achieve vertical sections of 50,80 or $100 \mu \mathrm{m}$ thickness. The slices were washed 3 times for 30 minutes in 1\% TX on a shaker at room temperature. Unspecific binding sites were blocked with $10 \%$ Normal Goat Serum (NGS, Invitrogen, Germany) in PBS 1\% TX for $1 \mathrm{~h}$ on a shaker at room temperature. The incubation in the primary antibody (rat anti-rabbit GABA, Sigma A2052 Germany, 1:400 in PBS 1\% TX plus sodium acid and NGS) took 6 days at $4^{\circ} \mathrm{C}$ on a shaker. Subsequently the brain was washed $6 \times 30$ minutes in PBS 1\% TX and thereafter incubated in the secondary antibody (goat anti-rabbit $\mathrm{Cy} 5, \mathrm{Cy} 3$ or Cy2,
1:200 in PBS 1\% TX plus $1 \mu$ l sodium acid) for 3 days on a shaker at $4^{\circ} \mathrm{C}$. The slices were washed for 30 minutes in PBS $1 \%$ TX and 5 times for 30 minutes in PBS at room temperature on a shaker. The slices were transferred in $60 \%$ glycerine in PBS and finally in $80 \%$ glycerine in PBS for confocal microscopy.

Additionally, antibody staining against glutamic acid decarboxylase (GAD, Millipore, Chemikon, United States, AB 1511, $\mathrm{GAD}_{67} \mathrm{GAD}_{65}$ ) were conducted. The procedure was the same as described for GABA except bovine serum albumin (BSA, Sigma, Germany) was used for blocking instead of NGS.

\section{Confocal microscopy}

For confocal microscopy (Leica TCS, Germany), the whole mounts were cleared in methylsalicylate or a mixture of benzyl alcohol and benzyl benzoate, as described previously and the slices in $80 \%$ glycerine in PBS. The whole mounts were embedded on metal microscope slides with a hole (diameter approximately $1 \mathrm{~cm}$ ) in the middle. A cover slip was fixed on top of the hole with super glue on the metal surface. The resulting cavity was big enough to allow for embedding of the whole bee brain.

Three lasers were used for the excitation of fluorescent dyes. Cy5 was excited with an $\mathrm{He} / \mathrm{Ne}$ laser at $633 \mathrm{~nm}$ wavelength, $\mathrm{Cy} 3$ as well as micro Ruby (Invitrogen, Germany) and tetramethylrodamine (TRITC) were excited with the He/Gre laser at $543 \mathrm{~nm}, \mathrm{Cy} 2$ and Lucifer yellow were excited with the $\mathrm{Ar} /$ $\mathrm{Kr}$ Laser at $488 \mathrm{~nm}$ wavelength. If one brain was excited with $633 \mathrm{~nm}$ as well as $543 \mathrm{~nm}$, sequential scanning was used in order to prevent cross excitation and bleaching. The PMT (photo multiplier tubes) was chosen between $400 \mathrm{~V}$ and $500 \mathrm{~V}$, and for intensity compensation in whole mounts, the PMT setting "linear by gain" was chosen. The offset was between 0 and -1 (background subtraction). For the scanning procedure, the beam expander 6 , the format $1024 \times 1024$ and the frame average $2-3$ was used. In the case of the $20 \times$ magnification water objective scanning steps of 1.5 to $2 \mu \mathrm{m}$ were used, with $10 \times$ magnification water and air objectives up to $2.5 \mu \mathrm{m}$ steps were used. Slices were scanned with $40 \times$ oil or glycerine objectives.

\section{D neuron reconstruction with Amira software (Visage} Imaging, Germany, version 4.1.1.)

The gray scale of the confocal slices was reconstructed with the skeleton tree tool (Amira). Two additional algorithms allowed for a thickness adjustment and straightening on the basis of the grey scale data of the scans and the skeleton tree. Background neuropile staining with Lucifer Yellow or the (less clear) Streptavidin background were used to reconstruct the neuropiles. In accordance with the standard atlas of the honeybee brain $^{24}$ the neuropiles were subdivided as follows: mushroom body with alpha lobe, beta lobe, pedunculus, medial and lateral calyx. The calyces were subdivided into lip, collar and basal ring. Additionally the protocerebrum with the central body was labelled. During the affine registration the labelled brain was 
coarsely adjusted to the standard brain. An affine transformation of the skeleton tree was necessary to adjust the neuron to the stretched neuropiles. During the last step, the elastic registration and transformation, a fine tuning to the standard brain was achieved.

This time consuming procedure was used for qualitatively excellent single neuron staining. In other cases the neurons have been visualized with less elaborate Amira tools, such as projection views, volrens, ortho slices or isosurfaces (Amira version 5.2).

\section{Spike sorting and statistics}

The raw intracellular recording channels were high pass filtered (high pass $220 \mathrm{~Hz}$, transition gap $150 \mathrm{~Hz}$, in most cases) with the program digital filters in Spike2 (CED, United Kingdom) in order to eliminate baseline fluctuations. This procedure was necessary in order to set a continuous threshold for the spike sorting (Spike2, CED, United Kingdom). The spike sorting result was confirmed by principal component analysis (Figure 4). Only separated scatterplots in the 3D space have been used as different units. Nevertheless, errors due to the sorting procedure cannot be excluded. Taking this inevitably occurring inaccuracy into account, the results of the spike sorting were called 'units'. For each set of units, extracted from one recording, the coefficient of correlation (Spearman's Rho, MATLAB 2010, Simulink) was calculated. Since long term effects have been described for PCT neurons ${ }^{20}$, unbinned spike times and binned spiketimes ( $1 \mathrm{~s}$ bins) were calculated and used for the correlation measurement. The correlation measurement of original (unbinned) spike times of different unit pairs was done in order to measure simultaneous spiking on the millisecond timescale. On the other hand, the comparison on the level of $1 \mathrm{~s}$ bins of unit spike times includes the correlation of spike trains (bursts). Only in the case of antisynchrony of spike trains on the longer timescale ( $1 \mathrm{~s}$ bins) can the effect be seen by eye (Figure 5). Antisynchrony on the short timescale (unbinned spike times) can also be achieved by the alternating occurrence of two units (unit1, unit2, unit1, unit2...etc.). A negative coefficient of correlation on the short timescale is the rule, rather than the exception. Contrarily, synchrony on the short time scale is a strong hint for the measurement of potentials of different cells. Potentials in a single (non-electrically coupled) cell should theoretically have a low coefficient of correlation (low synchrony) for original spike times on the short timescale (original spike times) due to the refractory period. In order to achieve maximal transparency, both values were calculated and summarized in Table 1 .

\section{Results}

Dye coupling of the recorded PCT neurons occurred in every successful Neurobiotin injection (Figure 3A-F, for examples) except one anatomically exceptional PCT neuron, with no arborizations in the calycal region of the mushroom body neuropile (Figure 2). Furthermore, the recordings of such dye

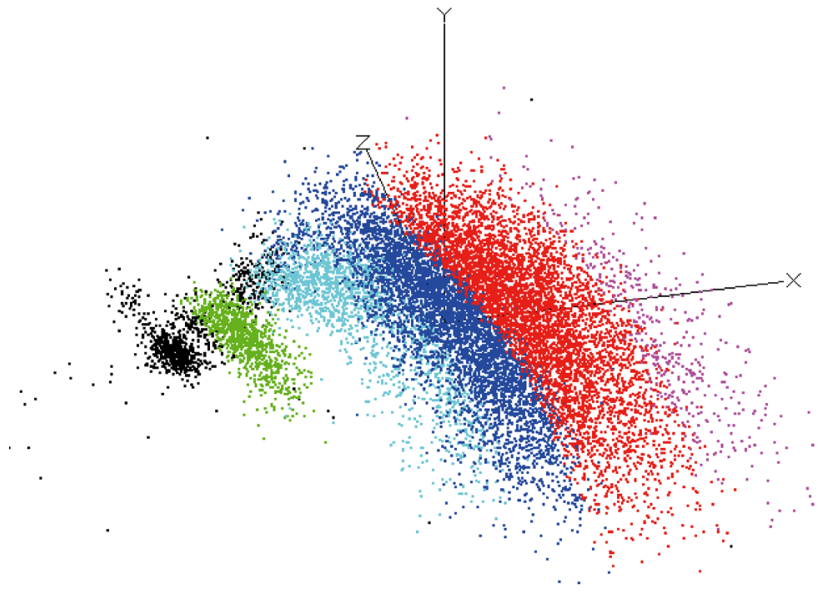

Figure 4 Principal component analysis for the three most variable components of the sorted spikes. The result (the example here is from bee n80108 with 5 units) was used in order to estimate the quality of the sorting result. Only spatially (3D) separated clusters have been recognized as different units.

coupled neurons revealed different spike shapes and amplitudes, which were sorted with a spike sorting algorithm (Spike2). In comparison to the single stained PCT neuron, mentioned beforehand, the spike amplitudes of dye-coupled PCT neurons were rather low (about $10 \mathrm{mV}$, Figures 6,7 ) and therefore called "spikelets". In order to account for possible errors due to the spike sorting procedure, the resulting sorted spikes are called units. The sorting results have been checked by a principal component analysis (example in Figure 4). Only anatomically identified PCT staining, without additionally stained non-PCT neurons, have been used for further analysis. Some neurons are completely stained (Figure 3A, C, E and F), others are identified by the characteristic soma clusters (Figure 3B and D). Multiple spike or spikelet shapes as well as antisynchronous activity patterns of these units were visible in all recorded, dye coupled units. In Figure 5, the spike sorting results of single electrode intracellular recordings are visualized as color maps for $1 \mathrm{~s}$ bins of spike times.

The coefficient of correlation was calculated in order to estimate the degree of synchrony for pairs of units on two time scales (Table 1). Direct spike times (short time scale, labelled s) and $1 \mathrm{~s}$ binned spike occurrences (longer time scale, labelled $\mathrm{l}$ ) were compared. The pairwise unit activity results are summarized in Table 1, including the coefficient of correlation (Spearman's Rho, labelled SR). Antisynchrony (negative coefficient of correlation) on both timescales occurred the most often (eight unit pairs). Seven unit pairs are synchronous (positive coefficient of correlation) on the short timescale (original spike times) but antisynchronous on the larger timescale ( $1 \mathrm{~s}$ bins). 

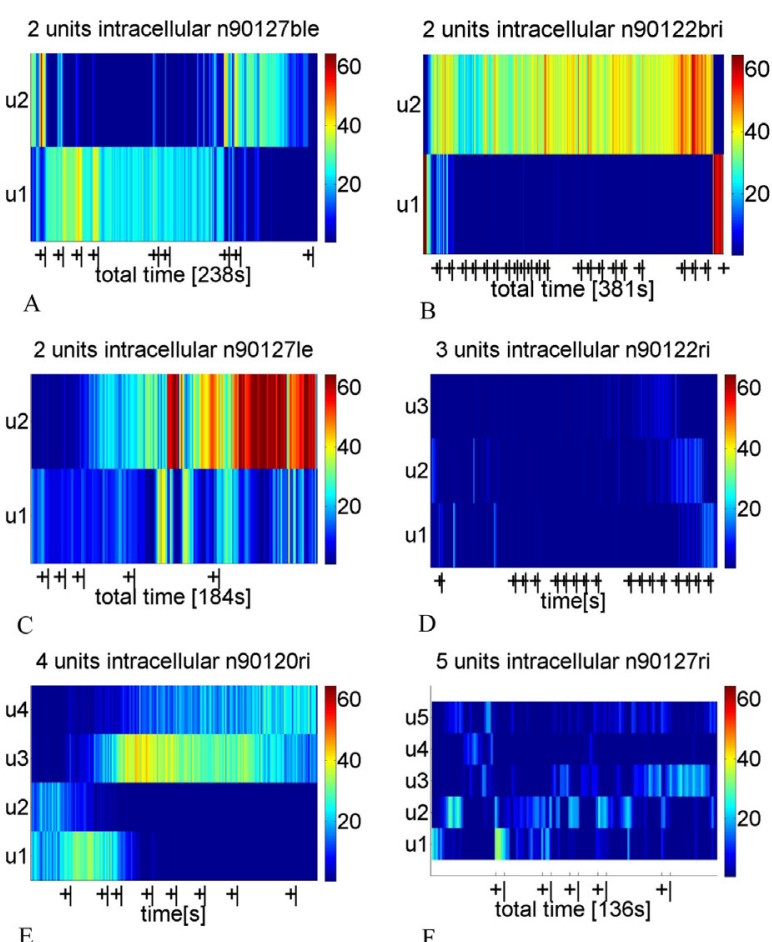

3 units intracellular n90122ri

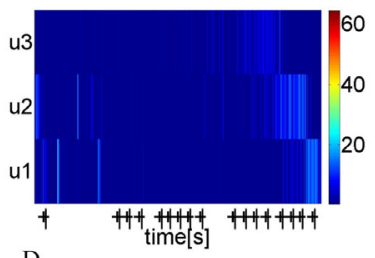

D

5 units intracellular n90127ri

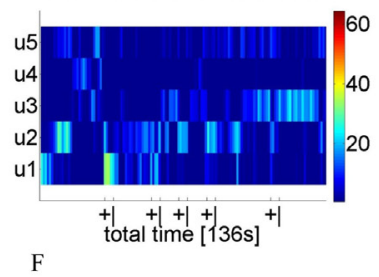

5 units intracellular n80108

$[\mathrm{Hz}]$

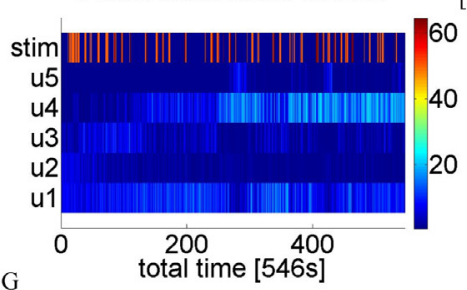

Figure 5 Antisynchronous activity patterns of dye coupled, intracellularly recorded and labelled Protocerebro Calycal Tract (PCT) neurons. The plus and minus signs below panels indicate the on- and offset of stimulations. In panel $\mathrm{G}$ the stimuli have been applied manually and are encoded as red stripes in the first row. Spike frequency ( $\mathrm{Hz}, 1 \mathrm{~s}$ bins) is color coded, as indicated in the color bar on the right side of each panel. Each panel contains 2 to 5 units. The following stimuli were applied: A [4x linalool (Lol), $2 \times$ octanone (8one), $2 \times$ heptanal (7al) and $1 \times$ hexanol (6ol)]; B [2x ipsiantennal sugar water (sipsi), contraantennal sugar water (scontra), tactile ipsiantennal stimulation (tipsi), $5 \times$ cineole (Col), $4 \times 8$ one, $2 \times 8$ one plus white light, white light, green light]; C [3x Lol and $2 \times$ Lol plus simultaneous presentation of white light]; D [2x sipsi, $1 \times$ scontra, $1 \times$ tipsi, $5 \times$ Col stimulation, $4 \times 8$ one stimulation, $2 \times 8$ one plus white light stimulation, $1 \times$ white light and $1 \times$ green light stimulation]; E [sipsi, $2 \times 60 l$, 6ol plus green light, green light, white light, 6ol, nonanone (9one)]; $\mathbf{F}[5 \times$ Lol]; $\mathbf{G}$ [2x blowing, $7 \times$ Col, $4 \times$ orange, $1 \times$ Lol, $6 \times$ geraniole, $3 \times$ Lol, $2 \times$ limonene, $3 \times 1$-nonanol, $7 \times$ clove, $2 \times$ orange, $1 \times$ blowing]. The stimulation events are shown in more detail in the peri stimulus time histograms in Figures 6, 7, 9 and 10.

Antisynchrony on the short but synchrony on the large time scale occurred in six cases. In eight cases, antisynchronous spiking for the short or long time scale is observed without a trend on the other timescale. In two cases, synchronous unit pairs

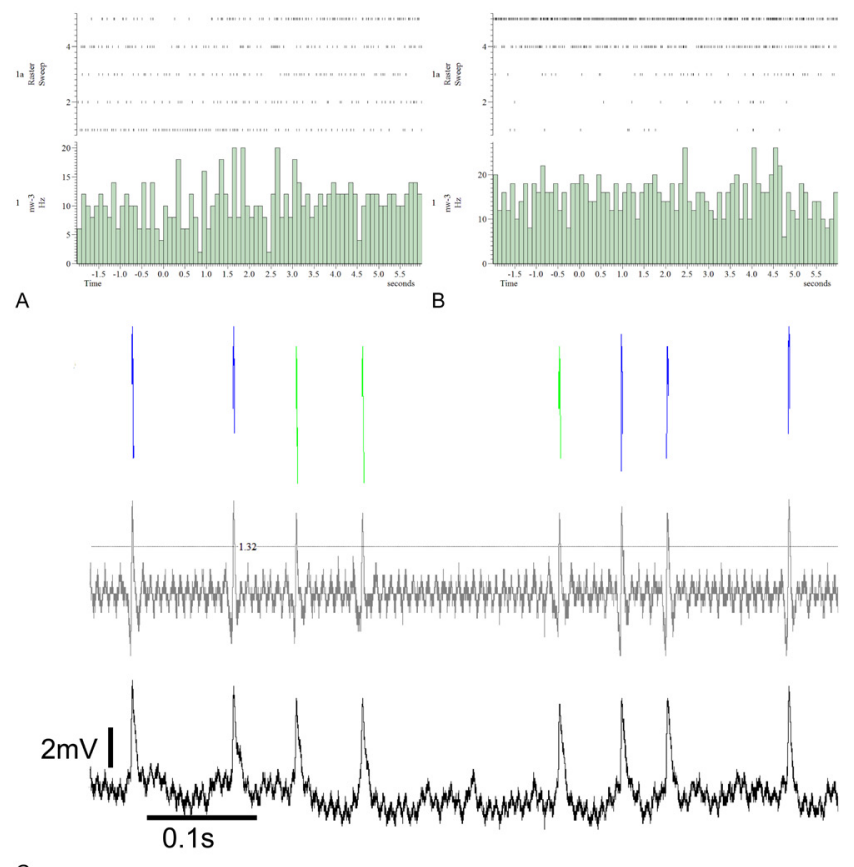

C

Figure 6 Peri stimulus time histograms (PSTH) for the recording in the left brain hemisphere of bee $\mathbf{n 9 0 1 2 7}$. The single traces 1-3 correspond to linalool stimulations, traces 4 and 5 to linalool and simultaneous white light. Neither unit1 (panel A) nor unit2 (panel B) show clear alterations in the spike rate due to the stimulation. To extract the units from the single electrode recordings, the raw data (panel $\mathbf{C}$, black trace) have been high pass filtered (panel $\mathbf{C}$, grey trace, high pass: $100 \mathrm{~Hz}$, transition gap: 50). Spikelets of unit1 are shown in blue, unit2 spikelets in green.

were observed on both the short and the longer timescale. In one case synchrony on the longer time scale occurred with no tendency for the short time scale.

Most PCT neurons can be colocalized with GABA and GAD${ }_{67} \mathrm{GAD}_{65}$ immunostaining, as seen in Figure 8 (at least one intracellulary labelled cell was colocalized within each immunostaining in four out of five cases. The five dye-coupled PCT intracellular recordings revealed 23 units). In Figures 8A-G, $\mathrm{GAD}_{67} \mathrm{GAD}_{65}$ and GABA stainings were done simultaneously. The comparison reveals similar colocalization patterns with the intracellular staining in the somata (Figures 8A-D) as well as axonal projections (Figure 8F and G). A colocalization for GABA but not $\mathrm{GAD}_{67} \mathrm{GAD}_{65}$ is seen in Figure $8 \mathrm{E}$. Figure $8 \mathrm{H}-\mathrm{L}$ shows GABA staining of the recorded PCT neurons at different cellular locations. Some somata show a colocalization with anti-GABA antibodies (Figure $8 \mathrm{H}$ ), others do not colocalize even though GABA stained somata are seen in near vicinity (Figure $8 \mathrm{~K}$ ). The same is true for other regions of the neuron, even in the same slice. As seen in Figure 8I and J, axonal segments, indicated by a grey arrowhead in Figure 8I, and the alpha exit point are clearly colocalized with GABA (colocalization in green), 


\begin{tabular}{|c|c|c|}
\hline Synchrony effects & $\begin{array}{l}\text { Total number } \\
\text { of pairwise } \\
\text { correlations }\end{array}$ & List of single measurements of Spearman's Rho (SR) \\
\hline Synchrony (s and I) & 2 & $\begin{array}{l}\text { N90120ri u1u2 SR s: } 0.31 \text { (Pval: } 6.9 \times 10^{-107} \text { ), I: } 0.78 \text { (Pval: } 4.5 \times 10^{-45} \text { ) } \\
\text { N80108ri u2u3 SR s: } 0.54 \text { (Pval: } 0 \text { ), I: } 0.18 \text { (Pval: } 2.5 \times 10^{-5} \text { ) }\end{array}$ \\
\hline $\begin{array}{l}\text { Synchrony (s), } \\
\text { Antisynchrony (I) }\end{array}$ & 7 & $\begin{array}{l}\left.\text { N90127ri u4u5 SR s: } 0.18 \text { (Pval: } 8 \times 10^{-8}\right) \text {, I: }-0.23 \text { (Pval: } 0.006 \text { ) } \\
\left.\text { N90127ri u1u3 SR s: } 0.3 \text { (Pval: } 2 \times 10^{-20} \text { ), I: }-0.57 \text { (Pval: } 4.2 \times 10^{-13}\right) \\
\text { N90127ri u1u5 SR s: } 0.36 \text { (Pval: } 1.6 \times 10^{-29} \text { ), I: }-0.25 \text { (Pval: } 0.003 \text { ) } \\
\left.\text { N90120ri u1u4 SR s: } 0.36 \text { (Pval: } 3.16 \times 10^{-146} \text { ), I: }-0.84 \text { (Pval: } 3.6 \times 10^{-56}\right) \\
\text { N80108ri u1u4 SR s: } 0.42 \text { (Pval: } 1.39 \times 10^{-237} \text { ), I: }-0.27 \text { (Pval: } 7 \times 10^{-11} \text { ) } \\
\left.\text { N80108ri u2u5 SR s: } 0.56 \text { (Pval: } 0 \text { ), I: }-0.18 \text { (Pval: } 1.6 \times 10^{-5}\right) \\
\text { N80108ri u3u5 SR s: } 0.28 \text { (Pval: } 2.18 \times 10^{-101} \text { ), I: }-0.54 \text { (Pval: } 8.28 \times 10^{-43} \text { ) }\end{array}$ \\
\hline $\begin{array}{l}\text { Antisynchrony (s), } \\
\text { Synchrony (I) }\end{array}$ & 6 & $\begin{array}{l}\text { N90127ri u1u2 SR s: - } 0.44 \text { (Pval: } 2.5 \times 10^{-44} \text { ), I: } 0.56 \text { (Pval: } 1.5 \times 10^{-12} \text { ) } \\
\text { N90127ri u2u5 SR s: }-0.71 \text { (Pval: } 9.6 \times 10^{-144} \text { ), I: } 0.19 \text { (Pval: } 0.024 \text { ) } \\
\text { N90127ri u3u5 SR s: }-0.16 \text { (Pval: } 10 \times 10^{-7} \text { ), I: } 0.23 \text { (Pval: } 0.007 \text { ) } \\
\left.\text { N90120ri u3u4 SR s: }-0.56 \text { (Pval: } 0 \text { ), I: } 0.31 \text { (Pval: } 4.44 \times 10^{-6}\right) \\
\text { N80108ri u1u3 SR s: }-0.5 \text { (Pval: 0), I: } 0.18 \text { (Pval: } 2.64 \times 10^{-5} \text { ) } \\
\text { N80108ri u4u5 SR s: }-0.47 \text { (Pval: } 1.17 \times 10^{-300} \text { ), I: } 0.56 \text { (Pval: } 7.1 \times 10^{-47} \text { ) }\end{array}$ \\
\hline $\begin{array}{l}\text { Antisynchrony (s), } \\
\text { Synchrony (I) }\end{array}$ & 8 & $\begin{array}{l}\left.\text { N90127ble SR s: }-0.53 \text { (Pval: } 2.77 \times 10^{-293} \text { ), I: }-0.61 \text { (Pval: } 5.1 \times 10^{-26}\right) \\
\text { N90127ri u2u4 SR s: }-0.58 \text { (Pval: } 5.2 \times 10^{-85} \text { ), I: }-0.42 \text { (Pval: } 3 \times 10^{-7} \text { ) } \\
\text { N90122bri u1u2 SR s: }-0.59 \text { (Pval: 0), I: }-0.23 \text { (Pval: } 5 \times 10^{-6} \text { ) } \\
\left.\text { N90120ri u2u3 SR s: }-0.59 \text { (Pval: 0), I: }-0.72 \text { (Pval: } 4.68 \times 10^{-35}\right) \\
\left.\text { N90120ri u1u3 SR s: }-0.75 \text { (Pval: 0), I: }-0.42 \text { (Pval: } 2.3 \times 10^{-10}\right) \\
\text { N80108ri u1u5 SR s: }-0.36 \text { (Pval: } 3.29 \times 10^{-168} \text { ), I: }-0.45 \text { (Pval: } 3.66 \times 10^{-28} \text { ) } \\
\text { N80108ri u2u4 SR s: }-0.65 \text { (Pval: 0), I: }-0.45 \text { (Pval: } 1.29 \times 10^{-28} \text { ) } \\
\text { N80108ri u3u4 SR s: }-0.75 \text { (Pval: 0), I: }-0.66 \text { (Pval: } 1.29 \times 10^{-68} \text { ) }\end{array}$ \\
\hline $\begin{array}{l}\text { No tendency (s), } \\
\text { Antisynchrony (I) }\end{array}$ & 4 & $\begin{array}{l}\left.\text { N90127ri u2u3 SR s: } 0.08 \text { (Pval: } 0.01) \text {, I: }-0.63 \text { (Pval: } 2.8 \times 10^{-16}\right) \\
\left.\text { N90127ri u1u4 SR s: }-0.1 \text { (Pval: } 0.003 \text { ), I: }-0.34 \text { (Pval: } 5 \times 10^{-5}\right) \\
\text { N90120ri u2u4 SR s: }-0.006 \text { (Pval: } 0.67) \text { NS, I: }-0.77\left(\text { Pval: } 2.66 \times 10^{-43} \text { ) }\right. \\
\left.\text { N90122ri u1u3 SR s: } 0.11 \text { (Pval: } 0.0018) \text {, I: }-0.3 \text { (Pval: } 1.6 \times 10^{-10}\right)\end{array}$ \\
\hline $\begin{array}{l}\text { Antisynchrony (s), } \\
\text { No tendency (I) }\end{array}$ & 4 & $\begin{array}{l}\text { N90127ri u3u4 SR s: }-0.33 \text { (Pval: } 4.7 \times 10^{-25} \text { ), I: } 0.07 \text { (Pval: } 0.45 \text { ) NS } \\
\text { N90127le u1u2 SR s: }-0.75 \text { (Pval: 0), I: }-0.1 \text { (Pval: } 0.3 \text { ) } \\
\text { N90122ri u2u3 SR s: }-0.67 \text { (Pval: } 2.2 \times 10^{-110} \text { ), I: } 0.14 \text { (Pval: } 0.003 \text { ) } \\
\text { N80108ri u1u2 SR s: }-0.48 \text { (Pval: } 2.9 \times 10^{-310} \text { ), I: }-0.1 \text { (Pval: } 0.019 \text { ) }\end{array}$ \\
\hline $\begin{array}{l}\text { No tendency (s), } \\
\text { Synchrony (I) }\end{array}$ & 1 & N90122ri u1u2 SR s: -0.12 (Pval: $7.7 \times 10^{-4}$ ), I: 0.26 (Pval: $1.1 \times 10^{-8}$ ) \\
\hline
\end{tabular}

$\mathrm{N}$ : experimental series (bees), s: short timescale, I: long timescale, u: unit, SR: Spearman's Rho, NS: not statistically significant.

whereas dendritic regions in the alpha-lobe exhibit less colocalized regions. The white arrows in panel I point towards tiny green patches of colocalization.

Multimodality, as has been previously described for PCT neurons ${ }^{20}$, can be confirmed by the data, presented here. The two units in Figure 9 are responding antagonistically towards the presentation of linalool and octanone. In the case of heptanal presentation, both units decrease their spike rate. Unit2 in panel $\mathrm{B}$ has a generally higher signal to noise ratio in comparison to unit 1 in panel A with high spontaneous activity. Not all recorded PCT neurons responded to odor stimulation, as shown in Figure 6A and B. Unit2 in panel B increases its spontaneous spike rate continuously over stimulation trials. The original recording is shown in panel C (black trace). The high pass filtered trace is indicated in grey as well as the sorted unit 1 in blue and unit 2 in green.
Five units were extracted via spike sorting in the right brain hemisphere in bee n90127 (Figure 7F). Unit1 (Figure 7A), unit2 (Figure 7B) and unit5 (Figure 7E) increased their firing rate during the odor stimulation $(5 \times$ linalool). The unit 1 response, with a phasic and a tonic component, decreases from trial 1-5 (Figure 7A). Unit2 also had a phasic and tonic component but the response strength was more or less constant along trials with an additional peak in spike rate for the offset of stimulation (Figure 7B). Unit5's response increased in strength from trial $1-5$ and is purely phasic (Figure 7E). The response of unit 3 to linalool stimulation is seen as a decrease in spike rate. This becomes more pronounced from trial $1-5$, because the spontaneous spike rate before and after the stimulation increases (Figure 7C). Unit4 did not respond to the odor presentations (Figure 7D). Unit4 activity decreases after the onset of odor presentations and is restricted to periods where the other units show decreased spiking activity (Figure 7D). Figure 10 


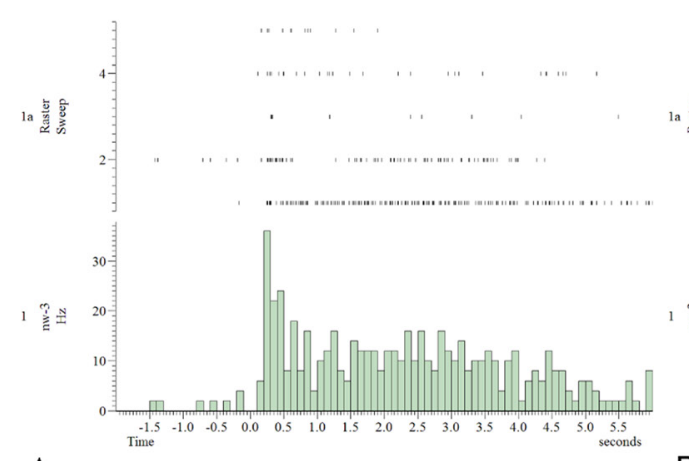

A

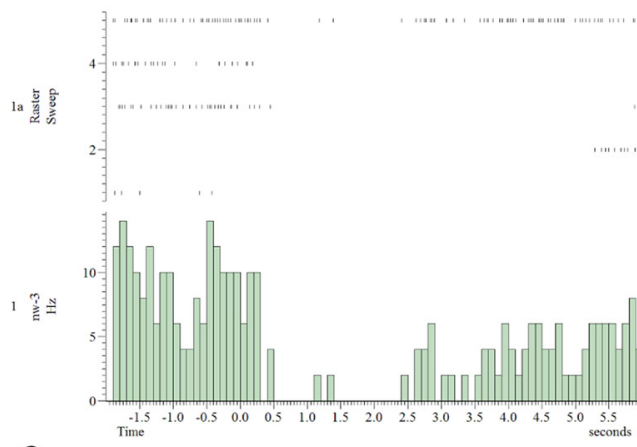

C

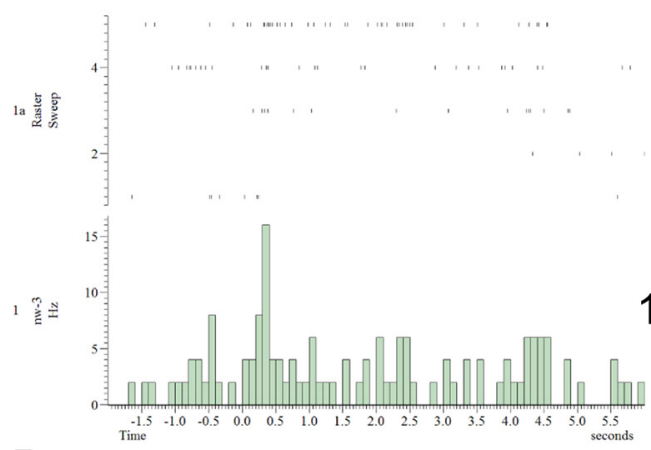

$\mathrm{E}$

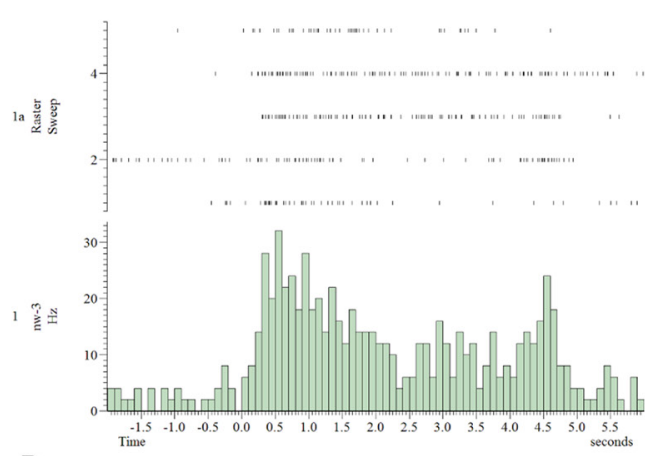

B

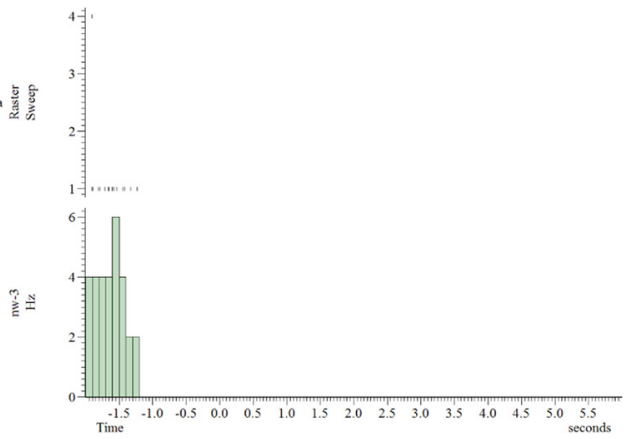

D
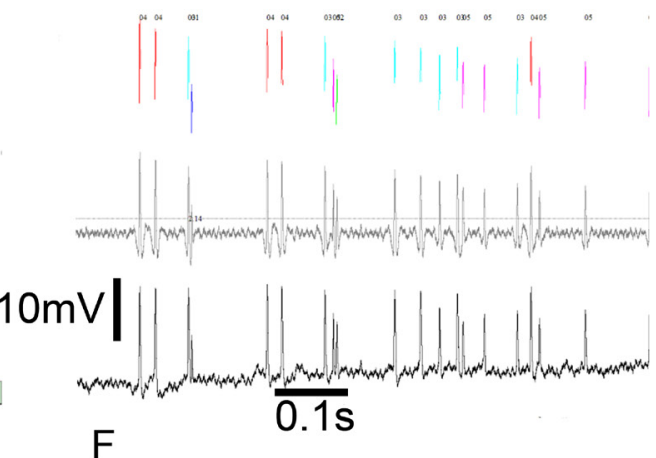

Figure 7 Peri stimulus time histograms (PSTH) for the recording of feedback neurons in the right brain hemisphere of bee n90127. Unit1 (panel A), unit2 (panel B) and unit5 (panel E) show an increase in the firing rate during stimulation with linalool. Unit3 firing rate is decreased during linalool application (panel C). Unit4 has only very short activity periods (panel D). Interestingly, the unit1 response, with a phasic and a tonic component, decreases from trials 1-5 whereas the unit5 response, with an almost pure phasic activity, increases from trials 1-5. Unit2 phasic-tonic activity is oscillating, but almost constant over trials. Unit3 activity increases from trials 1-5, but no spikes occur during the first 2 seconds of linalool stimulation. The raw data of the single electrode intracellular recording (panel $\mathbf{F}$, black trace) have been high pass filtered (panel $\mathbf{F}$, grey trace, high pass: $100 \mathrm{~Hz}$, transition gap: 50). The sorting result is shown in the colored channel.

gives an example of the occurrence of multisensory characteristics in some PCT neurons (Figure 10B). In contrast, unit1 in honeybee $\mathrm{n} 90122 \mathrm{~b}$ only responded to the first ipsiantennal sugar stimulation (Figure 10B). Spontaneous activity as well as response to stimuli increased in unit 1 but decreased in unit2.

In order to estimate the spike sorting accuracy, a single stained PCT neuron, which probably had no gap junction connections, was analyzed in the same way as the other recordings.
This PCT neuron did not show any dendritic arborizations in the calyces of the mushroom body. Instead, the pedunculus was innervated (color coded in yellow and cyan for the lateral and median ipsilateral mushroom body, respectively; Figure 2). The spike sorting revealed two well separated units (Figure 11A). The blue unit occurs only at the very beginning of the recording, due to tickle induced noise, which is confirmed by a principal component analysis (Figure 11B). The uniformly shaped spikes are illustrated in Figure 11C. 

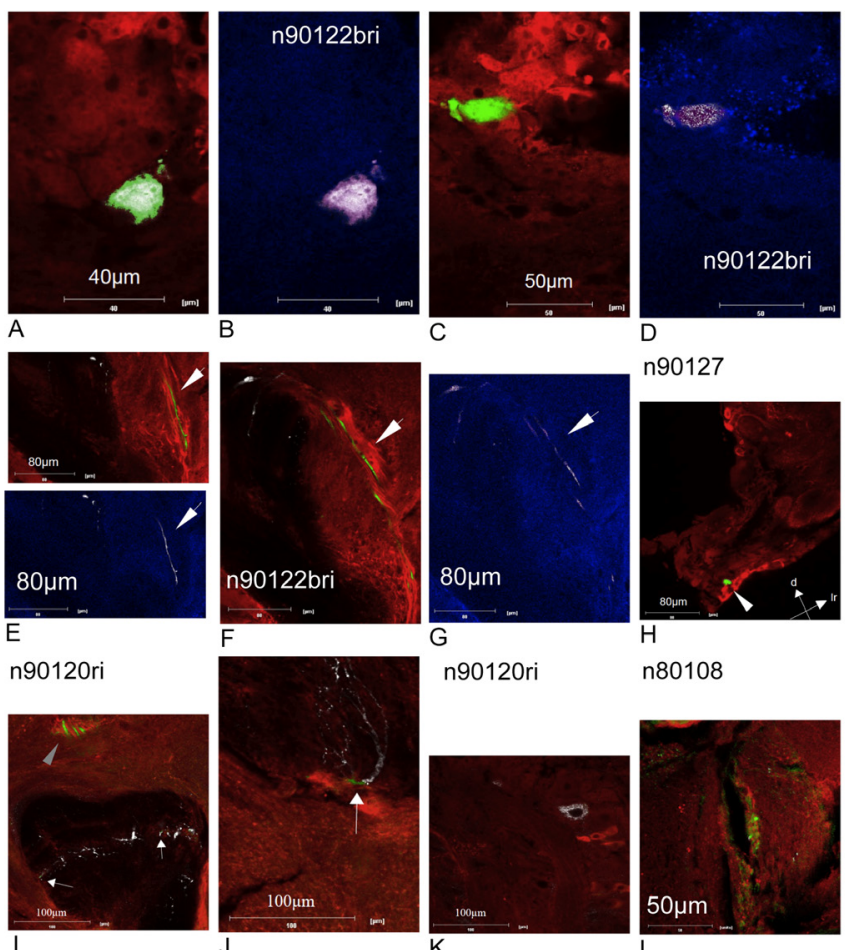

n90120ri

n90127

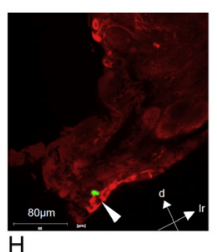

n80108
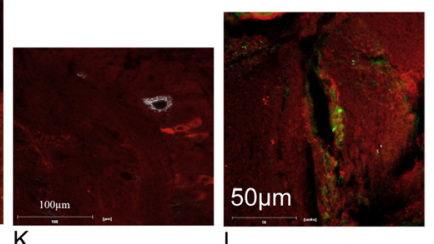

Figure 8 Immunostaining against GABA and $\mathrm{GAD}_{67} \mathrm{GAD}_{65}(400 \mathrm{x}$ magnification). A colocalization between the GABA (red) and $\mathrm{GAD}_{67} \mathrm{GAD}_{65}$ (blue) staining with the intracellularly injected dye is indicated in green and magenta, respectively. Somata as well as axonal projections (arrows in panels $\mathbf{E}$ and $\mathbf{F}$ ) in the intracellularly labelled Protocerebro Calycal Tract (PCT) neurons in the right brain hemisphere of bee $n 90122 b$ are colocalized with GABA and $\mathrm{GAD}_{67} \mathrm{GAD}_{65}$ (panels $\mathbf{A}-\mathbf{G}$ ). The GABA colocalization is not homogenously distributed (panels $\mathbf{H}-\mathbf{L}$ ), even in the same slice (panels I and $\mathbf{J}$, small patches of colocalization are highlighted by arrows). The regions for somata and axonal projections of PCT neurons within the bee brain are shown in Figures 1 and 11.

Confocal images of protocerebro calycal tract neurons colocalized with GABA and GAD immunostaining in Apis mellifera

120 Data Files

http://dx.doi.org/10.6084/m9.figshare.695020

Spike2 recordings of spike patterns in dye-coupled protocerebro calycal tract neurons in Apis mellifera

16 Data Files

http://dx.doi.org/10.6084/m9.figshare.695021

\section{Discussion}

This study investigated the occurrence of dye-coupling and synchrony effects of unit activity in PCT neurons.

Since single PCT neuron intracellular markings are sparse, especially with Neurobiotin $(286 \mathrm{mw})$ for Neurobiotin ${ }^{25}$, it can be assumed, that electrical coupling via gap junctions occurs in PCT feedback neurons. These observations have shown that single staining is more easily achieved with Lucifer Yellow, a bigger molecule than Neurobiotin $(457 \mathrm{mw}$ for Lucifer Yellow, $\left.{ }^{25}\right)$. In the following section, I will explain why gap junctions between subpopulations of PCT neurons are a parsimonious explanation for the phenomena observed in this investigation in contrast to the assumption of staining artefacts, which cannot be fully excluded. Cytoplasmic bridging seems to be unlikely, because only stable recordings have been used for analysis. Additionally, experiments with multiple neural staining other than PCT neurons have been excluded from analysis.

Let us assume that neighbouring PCT neurons are firing at their maximal rate. This might be a rare scenario but stable in terms of spike propagation. The respective refractory period of the neuron makes it impossible to propagate additional axo-axonal gap junction mediated potentials from the neighbouring cells. But what happens, if the firing rate of one PCT neuron is subthreshold with respect to the refractory period? Incoming signals from neighbouring PCT neurons, which are electrically coupled in their axonal regions, become more likely in this situation, because the cell, which is firing sparsely, is now susceptible to the propagation of laterally incoming potentials. Furthermore, the gap junction potentials are spreading bidirectionally through the invaded axon. In contrast, the spike propagation in the high frequency "potential source"-cell is unidirectional due to the refractory period. Spike coupling via gap junctions has some important implications for spike propagation including ultra fast information transfer ${ }^{26}$. Despite the observed asynchrony on the level of competing electrically coupled feedback neurons, the axo-axonal gap junctions lead to a high level of synchrony regarding the postsynaptic sites. But without feedback inhibition, this situation might be a dead end. Since the activity pattern of dye coupled ensembles consisted of fluctuating synchrony patterns on a relatively short timescale during an intracellular recording (up to 10 minutes), it is likely that a second assumption is realized in PCT neurons, that the gap junction mediated potentials are not only forward propagated (towards the calycal region) but also retrograde (towards the lobes). If the gap junction mediated, bidirectionally spreading spikes outperform the orthodromic spike initiation ${ }^{27}$ of the respective invaded PCT neuron, a retrograde signal becomes possible. Since the observed activity in the PCT is oscillatory ${ }^{20}$, the backward-feedback signal to neighbouring, electrically coupled neurons or their input sites in the lobes can be proposed as feedback inhibition of the invading cell and therefore a break against long lasting synchronization of coupled PCT neurons.

This mechanism is a realistic scenario since GABAergic cell interconnections have been observed in the mushroom body lobes ${ }^{21}$. The precise control of synchronized spike propagation in PCT neurons might be an important step in learning and memory formation in the honeybee brain. It has been shown 


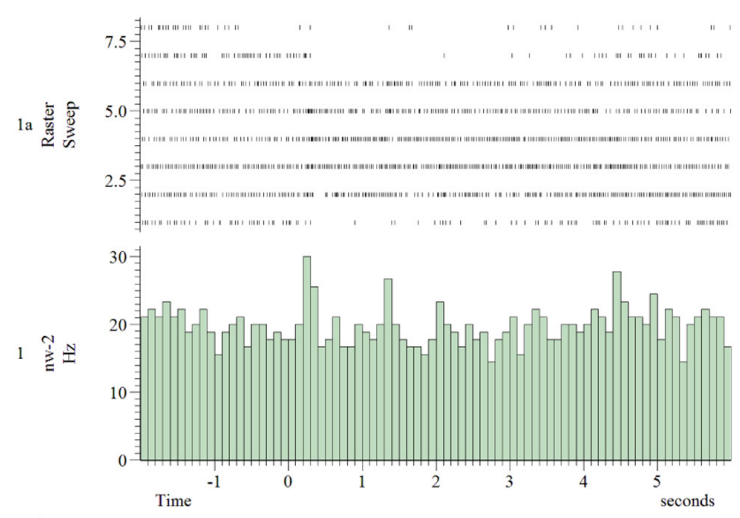

A

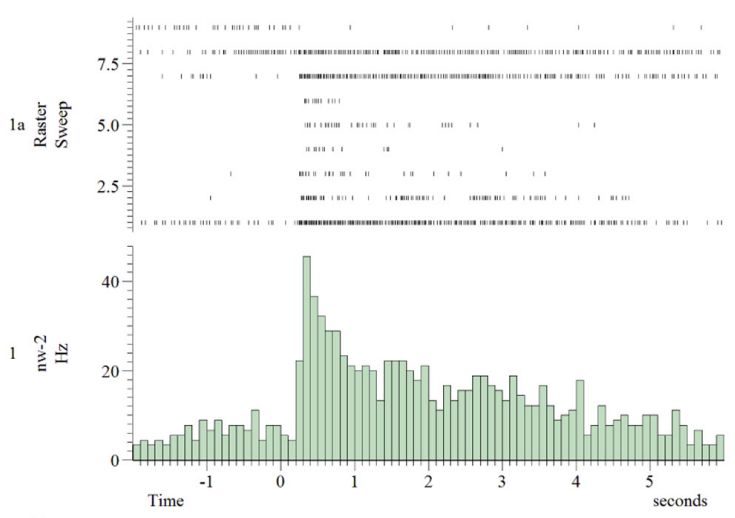

B

Figure 9 Peri stimulus time histograms (PSTHs) for $\mathbf{2}$ units in the left brain hemisphere of bee n90127b. Panel A shows the frequency and single traces for unit1. In panel B the same is shown for unit2. Unit2 (B) has a lower baseline firing rate and reaches higher peak spike rates due to stimulation. The order of stimulation (trace 1 to 9 in $\mathrm{B}$, trace 1 to 8 in $\mathrm{A}$, because unit1 was completely silent during the last (hexanol) stimulation) is as follows: $4 \times$ linalool, $2 \times$ octanone, $2 \times$ heptanal, $1 \times$ hexanol. The response to odorants is complementary in the two recorded feedback neuron units. A decrease in the firing rate of unit2 to linalool stimulation (the first 4 trials) is accompanied by an increase in unit1. Interestingly both units change their firing rate in a similar way in response to octanone and hexanol stimulation. Oscillatory response characteristics are seen in panel B, unit2, second trace, after linalool presentation. The oscillatory pattern is characterized by undulatory spiking activity after one sensory stimulation (raster plots).

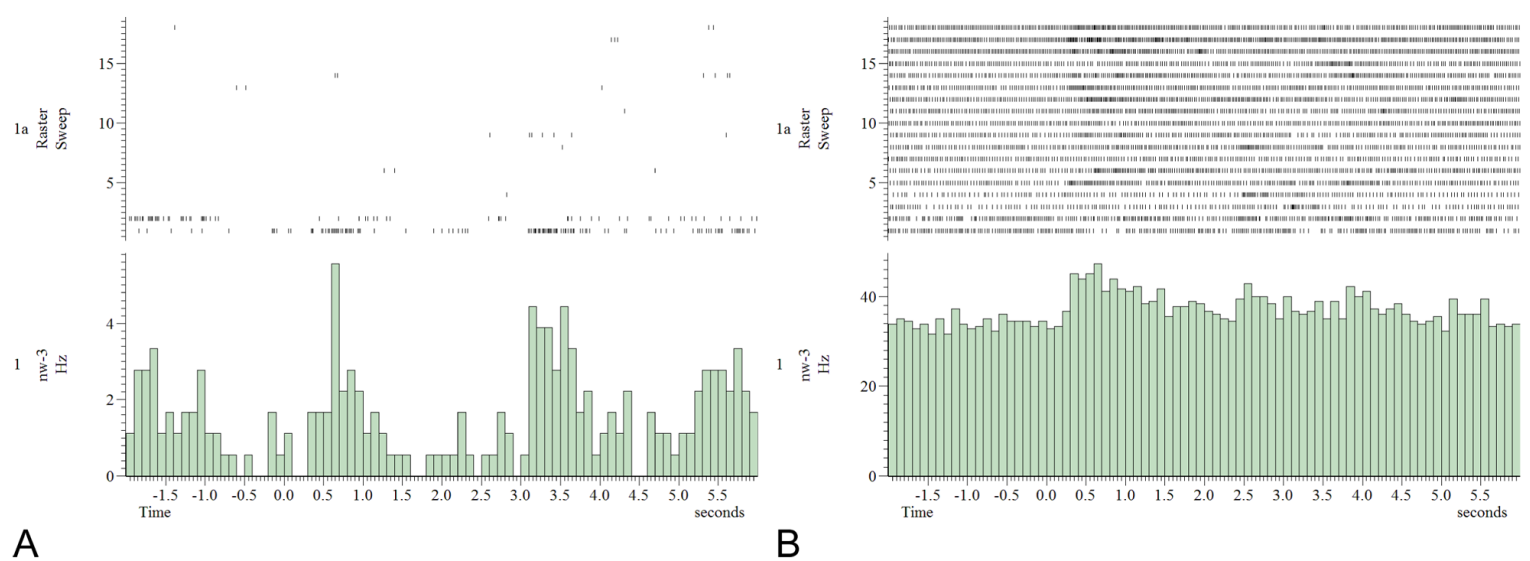

Figure 10 Peri stimulus time histograms (PSTH) for both recorded units in bee n90122b. Traces $1-18$ are the following stimuli: $2 \times$ ipsiantennal sugar water $\times$, contraantennal sugar water, tactile ipsiantennal stimulation, $5 \times$ cineole $\times$, $4 \times$ octanone $\times, 2 \times$ octanone plus white light $\times$, white light, green light. Unit1 $(\mathbf{A})$ is responding to the first ipsiantennal stimulation with sugar water. Subsequent unit1 spikes are very sparse. Unit2 (B) spike rate increases over trials, responding to light, sugar, odor and tactile stimuli. Oscillatory activity patterns are visible as well as variable latencies. 

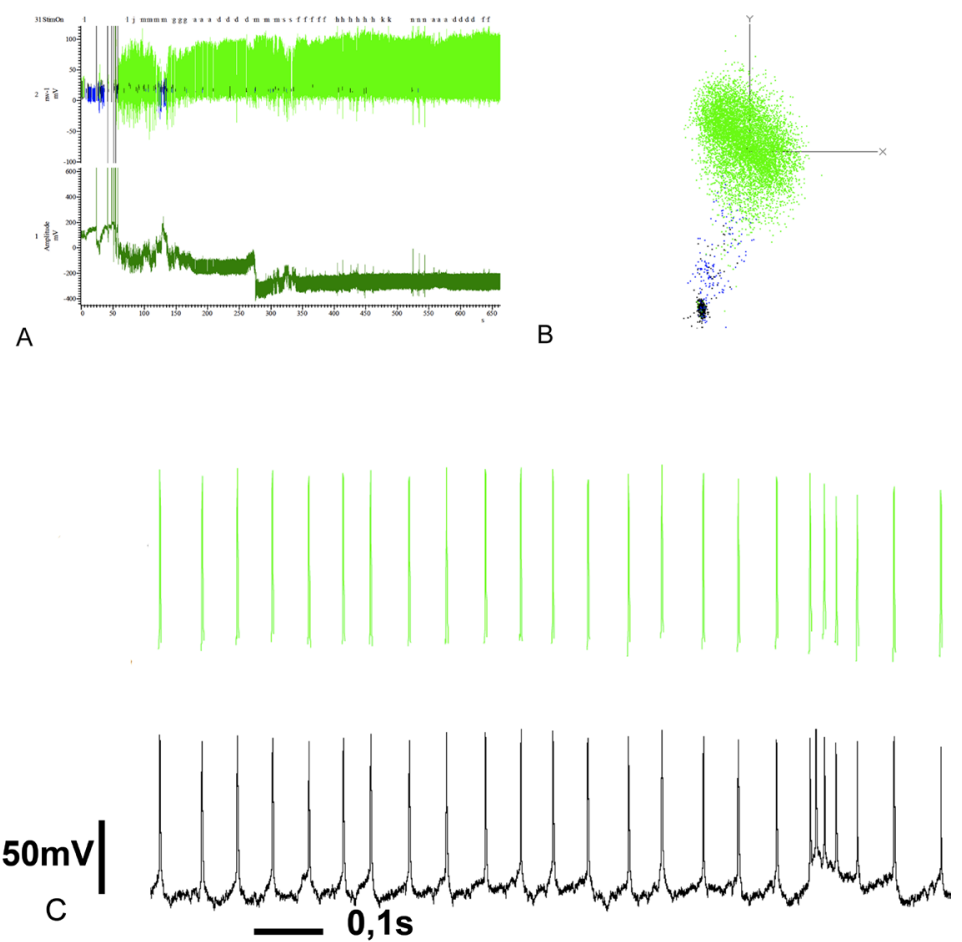

Figure 11 Physiology of a single stained Protocerebro Calycal Tract (PCT) neuron for comparison. The spike sorting reveals 1 unit (green, top trace, panel A). The blue unit is due to noise (the bottom trace is the original recording). Tickle events for cell penetration are seen as large artefacts at the left border of panel $\mathbf{A}$. In panel $\mathbf{B}$, the principal component analysis for the spike sorting is shown. In panel $\mathbf{C}$, original (black, bottom trace) and sorted spikes (green, top trace) are shown in order to estimate the similarity of spikes. The spikes occur regularly, despite some burst events, as seen on the right side of panel $\mathbf{C}$. The spike amplitude is much higher in comparison to the spikelets in the dye coupled PCT neurons (for example Figures. 7 and 8).

that synchrony-dependent processes occur in the bee brain ${ }^{28}$. Axo-axonal gap junctions are involved in the initiation of sharp wave/ripple events in the hippocampus ${ }^{26,29}$. Ripple events in turn have been shown to play an important role in the acquisition of memory in rodents ${ }^{30}$. It might be possible, that PCT neurons are a source of ripple-like local field potentials in insects. Another effect of axo-axonal gap junctions between feedback neurons might be an amplification effect regarding the output sites in the calycal region of the mushroom body, as it has been proposed to be involved in ectopic spike initiation (see Bucher and Goaillard for review ${ }^{31}$ ). This form of electrical connection between feedback neurons could be an alternative solution for ultrafast ${ }^{26}$, high frequency and broad band transmission to other cells without the requirement for giant fibres (as has been observed in locusts ${ }^{32}$.

The hypothesis of gap junctions between PCT neurons is further supported by the single stained exceptional PCT neuron without arborizations in the calycal region of the mushroom body, but instead has dendritic trees in the ipsilateral pedunculi (Figure 2). The spike sorting results in one unit (the second unit is clearly related to noise at the beginning of the recording, see Figure 11A). The gap junctions provide a mechanism for the coupling of subpopulations of neurons, which might be a source of synchrony for the information transferred to postsynaptic sites. Additionally, it has been shown that gap junctions can be uncoupled by rising intracellular calcium concentrations and $\mathrm{pH}$ changes ${ }^{33,34}$. These findings implicate that gap junction-coupled neurons cannot simply be regarded as functional units but rather that gap junctions provide complex mechanisms for neural plasticity.

In conclusion, the assumption of gap junctions between dye coupled feedback neurons in the mushroom body of bees provides an explanation for several features of these neurons, including oscillatory activity and multisensory response characteristics (Figures 6,7). The exciting question that arises is whether such oscillatory gap junction-mediated subnetworks can produce ripple-like events in insects. This is especially interesting since harp wave/ripples are associated with replay mediated consolidation of memory loads in rodents $s^{30}$. PCT neurons are potentially suited for the reactivation of sensory information, 
because they are part of complex synaptic aggregations in the mushroom body input regions, called microglomeruli ${ }^{19}$. It has been shown that Picrotoxin (a GABA A receptor blocker) injection in the honeybee brain led to reduced odor discrimination while learning ability per se was not affected. Furthermore, Picrotoxin application led to desynchronization between local field potential oscillations in the antennal lobe and the firing pattern of projection neurons ${ }^{35}$. The exact mechanisms of the output level of PCT neurons remain elusive, because they depend on the targeting of PCT neuron synapses and whether these synapses are really purely GABAergic. In order to further determine the occurrence of gap junctions in mushroom body calyces it might be interesting to perform dual intracellular recordings in the near vicinity as has been done in the rat hippocampus ${ }^{36}$, simultaneous injection of Neurobiotin and a second dye incapable of passing the gap junctions and both recordings and staining after the blockade of gap junctions with carbenoxolone ${ }^{36}$ or comparative drugs.

\section{Competing interests}

No competing interests were disclosed.

\section{Grant information}

The author declared that no grants were involved in supporting this work.
1. Brown TH, Chapman PF, Kairiss EW, et al: Long-term synaptic potentiation. Science. (1988); 242: 724-8.

2. Buzsáki G: Neural Syntax: Cell Assemblies, Synapsembles, and Readers. Neuron. (2010); 68: 362-385.

3. Chow CC, Kopell N: Dynamics of Spiking Neurons with Electrical Coupling. Neural Comput. (2000); 12: 1643-1678.

4. Cuntz H, Haag J, Forstner F, et al: Robust coding of flow-field parameters by axo-axonal gap junctions between fly visual interneurons. Proc Natl Acad Sci U S A. (2007); 104: 10229-10233.

5. Mercer A: Electrically coupled excitatory neurones in cortical regions. Brain Res. (2012); 1487: 192-197.

6. Hameroff S: How quantum brain biology can rescue conscious free will. Front Integr Neurosci. (2012); 6: 93.

7. Menzel R, Erber J, Masuhr T: learning and memory in the honeybee. In: Barton Browne L (ed) Experimental analysis of insect behaviour. Springer Verl, Berlin, Heidelberg, New York, (1974); pp195-217.

8. Erber J, Masuhr T, Menzel R: Localization of short-term memory in the brain of the bee, Apis mellifera. Physl Entomol. (1980); 5: 343-358.

9. Witthöft W: Absolute Anzahl und Verteilung der Zellen im Hirn der Honigbiene. Z Morphol Tiere. (1967); 61: 160-184.

10. Schürmann FW: Bemerkungen zur Funktion der Corpora pedunkulata im Gehirn der Insekten aus morphologischer Sicht. Exp Brain Res. (1974); 19: 406-432.

11. Mobbs PG: The brain of the honeybee Apis mellifera. I. The connections and spatial organization of mushroom bodies. Phil Trans R Soc Lond B. (1982); 298: 309-354.

12. Kenyon FC: The brain of the bee. A preliminary contribution to the morphology of the nervous system of the arthropoda. J Comp Neurol. (1896); 6: 133-210.

13. Rybak J, Menzel R: Anatomy of the mushroom bodies in the honey bee brain: the neuronal connections of the alpha-lobe. J Comp Neurol. (1993); 334: 444-465.

14. Goll W: Strukturuntersuchungen am Gehirn von Formica. $Z$ Morphol Oekol Tiere. (1967); 59: 143-210.

15. Schürmann FW: Über die Struktur der Pilzkörper des Insektengehirns III. Die Anatomie der Nervenfasern in den Corpora Pedunculata bei Acheta domesticus L (Orthoptera): Eine Golgi Studie. Z Zellforsch. (1973); 145: 247-285.

16. Schildberger K: Local interneurons associated with the mushroom bodies and the central body in the brain of Acheta domesticus. Cell Tissue Res. (1983); 230: 573-586.
17. Strausfeld NJ: Atlas of an insect brain. Springer, Berlin, Heidelberg, New York (1976).

18. Bicker G, Schäfer S, Kingan TG: Mushroom body feedback interneurons in the honeybee show GABA-like immunoreactivity. Brain Res. (1985); 360: 394-397.

19. Ganeshina O, Menzel R: GABA-Immunoreactive Neurons in the Mushroom Bodies of the Honeybee: An Electron Microscopic Study. J Comp Neurol. (2001); 437: 335-349.

20. Gronenberg W: Anatomical and physiological properties of feedback neurons of the mushroom bodies in the bee brain. Exp Biol. (1987); 46: 115-125.

21. Leitch B, Laurent G: GABAergic Synapses in the Antennal Lobe and Mushroom Body of the Locust Olfactory System. J Comp Neurol. (1996); 372: 487-514

22. Schürmann FW, Frambach I, Elekes K: GABAergic synaptic connections in mushroom bodies of insect brains. Acta Biol Hung. (2008); 59: 173-181.

23. Grünewald B: Physiological properties and response modulations of mushroom body feedback neurons during olfactory learning in the honeybee, Apis mellifera. J Comp Physiol A. (1999); 185: 565-576.

24. Brandt R, Rohlfing T, Rybak J, et al: Three-dimensional averageshape atlas of the honeybee brain and its applications. J Comp Neurol. (2005); 492: 1-19.

25. Penn AA, Wong RO, Shatz CJ: Neuronal Coupling in the Developing Mammalian Retina. J. Neurosci. (1994); 14: 3805-3815.

26. Schmitz D, Schuchmann S, Fisahn A, et al: Axo-Axonal Coupling: A Novel Mechanism for Ultrafast Neuronal Communication. Neuron. (2001); 31: 831-840.

27. Weidner C, Schmidt R, Schmelz M, et al: Action potential conduction in the terminal arborisation of nociceptive C-fibre afferents. J Physiol. (2003); 547: 931-940.

28. Menzel R, Manz G: Neural plasticity of mushroom body-extrinsic neurons in the honeybee brain. J Exp Biol. (2005); 208: 43174332.

29. Traub RD, Draguhn A, Whittington MA, et al: Axonal Gap Junctions Between Principal Neurons: A Novel Source of Network Oscillations, and Perhaps Epileptogenesis. Rev Neurosci. (2002); 13: 1-30.

30. Girardeau G, Benchenane K, Wiener SI, et al: Selective suppression of hippocampal ripples impairs spatial memory. Nat Neurosci. (2009); 12: 1222-1223. 
31. Bucher D, Goaillardr JM: Beyond faithful conduction: short-term dynamics, neuromodulation, and long-term regulation of spike propagation in the axon. Prog Neurobiol. (2011); 94: 307-346.

32. Papadopoulou M, Cassenaer S, Nowotny T, et al: Normalization for Sparse Encoding of Odors by a Wide-Field Interneuron. Science. (2011); 332: 721-725.

33. Baux G, Simonneau M, Tauc L, et al: Uncoupling of electrotonic synapses by calcium. Proc Natl Acad Sci U S A. (1978); 75: $4577-4581$
34. Skeberdis VA, Rimkute L, Skeberdyte A, et al: $\mathrm{pH}$-dependent modulation of connexin-based gap junctional uncouplers. J Physiol. (2011); 589: 3495-4506.

35. Stopfer M, Bhagavan S, Smith BH, et al: Impaired odour discrimination on desynchronization of odour-encoding neural assemblies. Nature. (1997); 390: 70-74.

36. Mercer A, Bannister AP, Thomson AM: Electrical coupling between pyramidal cells in adult cortical regions. Brain Cell Biol. (2006); 35: 13-27. 


\section{Peer review discontinued Peer review at F1000Research is author-driven. Currently no reviewers are being invited. What does this mean?}

\section{Version 1}

Reviewer Report 12 July 2013

https://doi.org/10.5256/f1000research.1365.r1058

(C) 2013 Yanovsky Y. This is an open access peer review report distributed under the terms of the Creative Commons Attribution License, which permits unrestricted use, distribution, and reproduction in any medium, provided the original work is properly cited.

\section{Yevgenij Yanovsky \\ University of Heidelberg, Heidelberg, Germany}

The data presented are of general and particular interests. All experiments have been properly done, and data were carefully analysed. However, the text in its present condition is difficult for comprehension and should be rearranged for better understanding.

Title is appropriate.

The Abstract should be rearranged to exclude discussion-like elements.

Introduction is too long and could be shortened at least to one-half of the present size. Everything concerning figures should be excluded from Introduction and moved to Results.

Minor point: Description of Fig. 3 occurred in text before that for Fig. 2.

Materials and methods. This part of manuscript is also too long. It might be helpful to describe here only main or originally built methods giving commonly used as references. Again, part of the text concerning figures should be put into Results.

Results. Experimental results would be better put in a more straight forward way, in support of the main line of study: morphological and immunostaining data showing die-coupling for GABA-ergic PCT neurons; spontaneous and evoked activity of these cells in aspect of de/synchronization as an evidence for gap junction mechanisms in the network. Pharmacological tests for gap junction connectivity would be very desirable.

Minor point: Fig. 2 could be combined with Fig. 11.

Minor point: Conclusions are justified on the basis of results.

Competing Interests: No competing interests were disclosed.

I confirm that I have read this submission and believe that I have an appropriate level of expertise to confirm that it is of an acceptable scientific standard. 
The benefits of publishing with F1000Research:

- Your article is published within days, with no editorial bias

- You can publish traditional articles, null/negative results, case reports, data notes and more

- The peer review process is transparent and collaborative

- Your article is indexed in PubMed after passing peer review

- Dedicated customer support at every stage

For pre-submission enquiries, contact research@f1000.com 